\title{
Critical behaviour of the Rouse model for gelling polymers
}

\author{
Peter Müller \\ E-mail: Peter.Mueller@physik.uni-goettingen.de \\ Institut für Theoretische Physik, Georg-August-Universität, D-37077 Göttingen, \\ Germany
}

Submitted to: J. Phys. A: Math. Gen. (in press)

\begin{abstract}
It is shown that the traditionally accepted "Rouse values" for the critical exponents at the gelation transition do not arise from the Rouse model for gelling polymers. The true critical behaviour of the Rouse model for gelling polymers is obtained from spectral properties of the connectivity matrix of the fractal clusters that are formed by the molecules. The required spectral properties are related to the return probability of a "blind ant"-random walk on the critical percolating cluster. The resulting scaling relations express the critical exponents of the shear-stress-relaxation function, and hence those of the shear viscosity and of the first normal stress coefficient, in terms of the spectral dimension $d_{s}$ of the critical percolating cluster and the exponents $\sigma$ and $\tau$ of the cluster-size distribution.

PACS numbers: 64.60.Ak, 64.60.Ht
\end{abstract}

\section{Introduction and result}

During a chemical gelation reaction the crosslinking of (macro-) molecules leads to the formation of permanent clusters of very different shapes and sizes. In the absence of a macroscopically large cluster such a system acts like a liquid (sol). Otherwise one deals with an amorphous solid state (gel). Hence, the sol-gel or gelation transition appears as a percolation transition [1-4], which is driven by crosslink concentration.

Gelling liquids exhibit striking mechanical properties which have been continuously studied over the years by experiments [5-12], theories [13-24] and simulations [25,26]. For example, when subjected to a homogeneous shear flow, distinct relaxation patterns are observed, which are due to the participation of many different excitation modes. More precisely, experiments suggest the scaling form $[2,4,6-8,12,16]$

$$
G(t) \sim t^{-\Delta} g\left(t / t^{*}\right) \quad \text { with } \quad t^{*}(\varepsilon) \sim \varepsilon^{-z}
$$

for the (shear-) stress-relaxation function in the sol phase for asymptotically long times $t$ and crosslink concentrations $c$ close to the critical point $c_{\text {crit }}$, i.e. for $\varepsilon:=\left(c_{\text {crit }}-c\right) / c_{\text {crit }} \ll$ 
1. The typical relaxation time $t^{*}$ diverges with a critical exponent $z>0$ for $\varepsilon \downarrow 0$. The scaling function $g$ is of order unity for small arguments so that one finds the algebraic decay $G(t) \sim t^{-\Delta}$ with a critical exponent $\Delta>0$ for $t \rightarrow \infty$ at the critical point $\varepsilon=0$. For large arguments $g$ decreases faster than any inverse power. Sometimes a stretched exponential has been proposed $[8,16]$ for $g$, implying the long-time asymptotics $-\ln G(t) \sim\left(t / t^{*}\right)^{\gamma}$ with some $0<\gamma<1$ in the sol phase.

Within linear viscoelasticity one finds $\eta=\int_{0}^{\infty} \mathrm{d} t G(t)$ for the zero-frequency viscosity and $\Psi_{1}=2 \int_{0}^{\infty} \mathrm{d} t t G(t)$ for the first normal stress coefficient [4,27]. The scaling form (1) of the stress-relaxation function determines their critical divergencies $\eta \sim \varepsilon^{-k}$ and $\Psi_{1} \sim \varepsilon^{-\ell}$ as $\varepsilon \downarrow 0$ by the scaling relations $[4,24]$

$$
k=z(1-\Delta) \quad \text { and } \quad \ell=z(2-\Delta)=k+z .
$$

Thus, it suffices to know any two of the four critical exponents $\Delta, z, k$ and $\ell$.

The Rouse model is one of the most basic models for polymer dynamics. By definition [28-30], it is a bead-spring model for linear polymer chains with no other interactions among monomers (i.e. beads) than connectivity along the chain, as accounted for by harmonic springs. Moreover, each bead behaves as a Brownian particle in that it experiences a friction force and a thermal noise force. Despite its simplicity the Rouse model has provided a much valued description of linear polymer chains over the last fifty years. Its generalization from linear chains to arbitrarily connected clusters of monomers is straightforward and will be recalled in Section 2. Static and dynamic properties of such Rouse clusters have been discussed, for example, in [15,31-34], and recent applications to gelling polymer melts can be found in [18,20-24]. More than twenty years earlier, de Gennes was the first who aimed at a description of the gelation transition in terms of the Rouse model. Guided by scaling arguments, he claimed the relation $k=2 \nu-\beta$ for the critical exponent of the viscosity [14]. Here, the exponent $\nu$ governs the divergence of the correlation length and $\beta$ the growth of the gel fraction. Using this result, it was also argued $[6,7,10,16,35]$ that $\Delta=d \nu /(d \nu+k)$, where $d$ is the space dimension. Inserting the numerical values for three-dimensional bond percolation $[36,37]$, as is generally accepted, gives $k \approx 1.35$ and $\Delta \approx 0.66$. These results have become known as the "Rouse values" for the critical exponents and are commonly quoted in the literature, see e.g. the reviews [1-4].

In contrast, the exact solution of the Rouse model for gelling polymers in $[20,21]$ leads to

$$
k=\left(1-\tau+2 / d_{s}\right) / \sigma
$$

and thus disproves the earlier scaling arguments - and hence the Rouse value - for $k$ mentioned above. Here $d_{s}$ denotes the spectral dimension [36-38] of the incipient percolating cluster, and the exponents $\sigma$ and $\tau$ are related to the cluster-size distribution, as defined in (12) below. The derivation of (3) in [20,21] exploits a correspondence between Rouse clusters and an electrical resistor network, which rests on the linearity of Hooke's law and Ohm's law. This correspondence is exact. It differs from de Gennes' superconductor/conductor-network analogy [39], as can be seen most clearly 
Critical behaviour of the Rouse model for gelling polymers

Table 1. Critical exponents for a gelling Rouse melt based on (4) and (2).

\begin{tabular}{lllll}
\hline & $\Delta=d_{s}(\tau-1) / 2$ & $z=2 /\left(d_{s} \sigma\right)$ & $k=z(1-\Delta)$ & $\ell=z(2-\Delta)$ \\
\hline $2 \mathrm{D}$ & 0.70 & 3.8 & 1.2 & 5.0 \\
$3 \mathrm{D}$ & 0.79 & 3.3 & 0.71 & 4.1 \\
$\mathrm{MF}$ & 1 & 3 & 0 & 3 \\
\hline
\end{tabular}

by comparing the resulting numerical values for $k$ in two space dimensions [20]. Unfortunately, the approach of $[20,21]$, which led to (3), does not seem to be suitable for a computation of the other exponents $\Delta, z$ or $\ell$-except for the case where the statistical ensemble of the clusters corresponds to mean-field percolation [22-24]. Hence, the authors of [24] had to use the numerically estimated value $\Delta \approx 0.83$, which was obtained in [22] for the Rouse model for gelling polymers with clusters distributed according to three-dimensional percolation.

The present Paper fills this gap. We present a simple analytical calculation which determines both $\Delta$ and $z$-and hence also $k$ and $\ell$ by (2) - for Rouse clusters distributed according to $d$-dimensional percolation. This will be achieved by a well-known relation between the dynamics of Rouse clusters and a "blind ant"-random walk on percolation clusters $[15,36,37]$. In particular, we will express the stress-relaxation function $G$ in terms of the return probability of such a random walk. In this way we will deduce the scaling relations

$$
\Delta=\frac{d_{s}}{2}(\tau-1) \quad \text { and } \quad z=\frac{2}{d_{s} \sigma}
$$

for the microscopic Rouse model for gelling polymers. Of course, (4) and (2) are consistent with existing exact results, that is, equation (3) for $k$ [20,21] and the meanfield values for $\Delta, z$ and $\ell$ [22-24]. Table 1 summarizes the exact values of the critical exponents pertaining to the Rouse model for gelling polymers with clusters distributed according to two- and three-dimensional lattice-bond percolation, respectively meanfield percolation.

The result for $\Delta$ in (4) differs from the relation $\Delta=d \nu /(d \nu+k)$ that has grown out of the scaling arguments mentioned above. Hence, it is not only the "Rouse value" for $k$ but also the one for $\Delta$ that has been ascribed incorrectly to the Rouse model for gelling polymers.

The rest of the Paper is organized as follows. After recalling the Rouse model for gelling polymers in Section 2, we will derive the scaling relations (4) within this model in Sections 3 to 6 . Section 7 contains a discussion and an outlook.

\section{Rouse model for gelling polymers}

In order to set up the notation we recall the standard definition [15, 29, 30, 32-34] of the Rouse model for clusters of monomers with an arbitrary topology. Previous applications to gelling polymer melts can be found in [18,20-24]. We consider $N$ point-like monomers 
with positions $\boldsymbol{R}_{i}(t), i=1, \ldots, N$, in $d$-dimensional space, $d \geq 2$. Their motion is constrained by $M$ randomly chosen, permanent, harmonic crosslinks which connect the pairs of monomers $\left(i_{e}, j_{e}\right), e=1, \ldots, M$, and give rise to the potential energy

$$
U:=\frac{d}{2 a^{2}} \sum_{e=1}^{M}\left(\boldsymbol{R}_{i_{e}}-\boldsymbol{R}_{j_{e}}\right)^{2}=: \frac{d}{2 a^{2}} \sum_{i, j}^{N} \Gamma_{i j} \boldsymbol{R}_{i} \cdot \boldsymbol{R}_{j} .
$$

Here, we have chosen units such that the inverse temperature $\left(k_{\mathrm{B}} T\right)^{-1}$ is equal to one, and the fixed length $a>0$ serves as an inverse crosslink strength. The second equality in (5) introduces the random $N \times N$-connectivity matrix $\Gamma$, which encodes all properties of a given crosslink realisation. The simple relaxational dynamics

$$
0=-\zeta\left[\frac{\mathrm{d}}{\mathrm{d} t} \boldsymbol{R}_{i}(t)-\boldsymbol{v}_{\mathrm{ext}}\left(\boldsymbol{R}_{i}(t), t\right)\right]-\frac{\partial U}{\partial \boldsymbol{R}_{i}}(t)+\boldsymbol{\xi}_{i}(t)
$$

governs the motion of the monomers in the externally applied shear flow $v_{\text {ext }}^{\alpha}(\boldsymbol{r}, t):=$ $\kappa(t) \delta_{\alpha, 1} r_{2}$, which is oriented along the 1-direction and involves the spatially homogeneous, time-dependent shear rate $\kappa(t)$. Here, Greek indices label Cartesian components, $\boldsymbol{r}=\left(r_{1}, \ldots, r_{d}\right)$ and $\delta_{\alpha, \beta}$ denotes the Kronecker symbol. A friction force with friction constant $\zeta$ applies to monomer $i$, if its velocity deviates from that of the surrounding shear flow. Moreover, the crosslinks exert the Hookean forces $-\partial U / \partial \boldsymbol{R}_{i}$, and the last contribution in (6) is due to a randomly fluctuating thermal-noise force $\boldsymbol{\xi}_{i}(t)$, obeying Gaussian statistics with zero mean and covariance $\overline{\xi_{i}^{\alpha}(t) \xi_{j}^{\beta}\left(t^{\prime}\right)}=2 \zeta \delta_{\alpha, \beta} \delta_{i, j} \delta\left(t-t^{\prime}\right)$. Here, $\delta(t)$ denotes the Dirac delta function. Given the homogeneous shear flow $\boldsymbol{v}_{\text {ext }}$ and any fixed configuration $\mathcal{C}:=\left\{\left(i_{e}, j_{e}\right)\right\}_{e=1}^{M}$ of permanent crosslinks, the equation of motion (6) is linear and can be solved exactly for every realisation of the thermal noise [21].

It remains to specify the statistical ensemble which determines the probabilistic occurrence of different crosslink configurations $\mathcal{C}$. Here we consider any bondpercolation ensemble which is amenable to a scaling description, does not distinguish any site (i.e. monomer), yields a fractal percolating cluster at criticality and where the maximum number of bonds (i.e. crosslinks) emanating from a given site is uniformly bounded. Besides certain versions of continuum percolation [40], this also includes bond percolation on the simple cubic lattice in $d$ dimensions and on the Cayley tree $[36,37]$. The statistical average over all crosslink configurations will be denoted by angular brackets $\langle\bullet\rangle$ and, when using this notation, we implicitly assume that the macroscopic limit $N \rightarrow \infty, M \rightarrow \infty, M / N \rightarrow c$ is carried out, too.

\section{Stress relaxation in the Rouse model for gelling polymers}

The crosslinks exert shear stress on the molecular clusters due to the externally applied shear flow. Following Kirkwood [29,30], the Cartesian components of the stress tensor are given in terms of a force-position correlation

$$
\sigma_{\alpha \beta}(t)=\lim _{t_{0} \rightarrow-\infty} \frac{\rho_{0}}{N} \sum_{i=1}^{N} \overline{\frac{\partial U}{\partial R_{i}^{\alpha}}(t) R_{i}^{\beta}(t)} .
$$


Here, $\rho_{0}$ denotes the density of monomers, and one has to insert the explicitly known [21] solutions $\boldsymbol{R}_{i}(t)$ of the Rouse equation (6) with some initial data $\boldsymbol{R}_{i}\left(t_{0}\right)$. Finally, the limit $t_{0} \rightarrow-\infty$ ensures that all transient effects stemming from the initial data will disappear. This yields the exact result

$$
\langle\boldsymbol{\sigma}(t)\rangle=G(0) \mathbf{1}+\int_{-\infty}^{t} \mathrm{~d} t^{\prime} G\left(t-t^{\prime}\right) \kappa\left(t^{\prime}\right)\left(\begin{array}{cccc}
2 \int_{t^{\prime}}^{t} \mathrm{~d} s \kappa(s) & 1 & 0 & \cdots \\
1 & 0 & 0 & \cdots \\
0 & 0 & 0 & \cdots \\
\vdots & \vdots & \vdots & \ddots
\end{array}\right)
$$

for the crosslink-averaged, macroscopic stress tensor, where $\mathbf{1}$ is the $3 \times 3$-unit matrix and

$$
G(t) \equiv G(t, \varepsilon):=\left\langle\frac{\rho_{0}}{N} \operatorname{Tr}\left[\left(1-E_{0}\right) \exp \left(-\frac{6 t}{\zeta a^{2}} \Gamma\right)\right]\right\rangle
$$

represents the stress-relaxation function $[21,24]$. Here, $\mathrm{Tr}$ is the trace and $E_{0}$ denotes the eigenprojector on the space of zero eigenvalues of $\Gamma$, which correspond to translations of whole clusters $[18,20,21]$. Within the simple Rouse model the zero eigenvalues do not contribute to stress relaxation because there is no force acting between different clusters. The only contribution to stress relaxation is due to deformations of the clusters.

\section{Cluster decomposition}

A crosslink configuration $\mathcal{C}$ can be decomposed into $K \equiv K(\mathcal{C})$ disjoint clusters, and we denote the number of monomers in its $k$ th cluster $\mathcal{N}_{k} \equiv \mathcal{N}_{k}(\mathcal{C})$ by $N_{k} \equiv N_{k}(\mathcal{C})$. The connectivity matrix $\Gamma(\mathcal{C})$ of the whole configuration is thus a block-diagonal sum of connectivity matrices $\Gamma\left(\mathcal{N}_{k}\right)$ of different clusters. Hence, when evaluating the trace in (9), one gets an additive contribution from every cluster. Upon sorting these contributions with respect to the number of monomers in the clusters and introducing the mean number density $\tau_{n}:=\left\langle N^{-1} \sum_{k=1}^{K} \delta_{N_{k}, n}\right\rangle$ of clusters with $n$ monomers-also known as cluster-size distribution-, we arrive at the cluster-decomposed form

$$
G(t, \varepsilon)=\sum_{n=1}^{\infty} n \tau_{n}(\varepsilon) G_{n}(t, \varepsilon)
$$

of the stress-relaxation function. Here, the mean stress-relaxation function of $n$-clusters

$$
G_{n}(t, \varepsilon):=\left\langle\frac{\rho_{0}}{n} \operatorname{Tr}\left[\left(1-E_{0}\right) \exp \left(-\frac{6 t}{\zeta a^{2}} \Gamma\right)\right]\right\rangle_{n}
$$

is defined in terms of the (conditional) average $\langle A\rangle_{n}:=\tau_{n}^{-1}\left\langle N^{-1} \sum_{k=1}^{K} \delta_{N_{k}, n} A\left(\mathcal{N}_{k}\right)\right\rangle$ for an observable $A$ over all clusters with $n$ monomers. The cluster-size distribution $\tau_{n}$ gives rise to the critical exponents $\sigma$ and $\tau$ through its scaling form

$$
\tau_{n}(\varepsilon) \sim n^{-\tau} f_{1}\left(n / n^{*}(\varepsilon)\right)
$$

for $n \gg 1$ and crosslink concentrations close to the critical point [36,37]. It involves the typical cluster mass $n^{*}(\varepsilon) \sim \varepsilon^{-1 / \sigma}$, which diverges for $\varepsilon \downarrow 0$. The scaling function $f_{1}$ 
decreases faster than any inverse power for large arguments, whereas for small arguments it approaches a non-zero, finite constant. It will turn out later that in order to calculate the critical exponents $\Delta$ and $z$ of $G$, it suffices to know $G_{n}$ at the gelation transition. For this reason we will only be concerned with critical percolation clusters in the next paragraph.

\section{Blind ant on critical percolation clusters}

Consider a random walker - coined "blind ant" by de Gennes [41] - that moves along a bond from one site to another in the same cluster at discrete time steps [36-38,42]. More precisely, let $b$ denote the maximum number of possible bonds which are allowed to emanate from a site in the percolation model under consideration. If the ant happens to visit site $i$ at time $s$, which is connected with $b_{i} \leq b$ bonds to other sites, then it will move with equal probability $1 / b$ along any one of the $b_{i}$ bonds within the next time step and stay at site $i$ with probability $1-b_{i} / b$. By the definition (5) of the connectivity matrix $\Gamma$ of the cluster, one has $\Gamma_{i i}=b_{i}$ for its diagonal matrix elements, $\Gamma_{i j}=-1$ if two different sites $i \neq j$ are connected by a bond and zero otherwise. Hence, the associated master equation for the ant's sojourn probability $p_{i}(s)$ for site $i$ at time $s$ reads

$$
p_{i}(s+1)=\left(1-\Gamma_{i i} / b\right) p_{i}(s)+\sum_{j \neq i}\left(-\Gamma_{i j} / b\right) p_{j}(s),
$$

which is equivalent to

$$
p_{i}(s+1)-p_{i}(s)=-b^{-1} \sum_{j} \Gamma_{i j} p_{j}(s) .
$$

Here the summation extends over all sites in the cluster. For long times $s \gg 1$, it is legitimate to replace the difference (quotient) on the left-hand side of (14) by a derivative. This yields the solution $p_{i}(s)=\left[\mathrm{e}^{-s \Gamma / b}\right]_{i i_{0}}$, which corresponds to the initial condition $p_{i}(0)=\delta_{i, i_{0}}$.

Next we consider $P^{(n)}(s):=\left.\left\langle p_{i_{0}}(s)\right\rangle_{n}\right|_{\varepsilon=0}$, the mean return probability to the starting point after time $s$, where the average is taken over all critical percolation clusters with $n$ sites. Likewise, $P^{(\infty)}(s)$ stands for the mean return probability on the incipient percolating cluster. Clearly, these definitions are independent of the starting point $i_{0}$, because on average there is no distinguished site by assumption. Thus we can also write

$$
P^{(n)}(s)=\left.\left\langle\frac{1}{n} \operatorname{Tr} \mathrm{e}^{-s \Gamma / b}\right\rangle_{n}\right|_{\varepsilon=0}
$$

for finite $n$. The decay of $P^{(\infty)}(s)$ for long times $s \gg 1$ is determined by the inverse number of distinct sites which the ant has visited up to time $s$ and, thus, by the spectral dimension $d_{s}$ of the incipient percolating cluster according to $[37,38,42]$

$$
P^{(\infty)}(s) \sim s^{-d_{s} / 2} .
$$

The fractal nature of percolation clusters at the critical point implies that clusters with $n \gg 1$ sites typically look like regions of $n$ connected sites that are cut out of the 
infinite cluster. Therefore we conclude $P^{(n)}(s) \sim s^{-d_{s} / 2}$ for times $1 \ll s \lesssim s_{n}$, where $[37,38,42]$

$$
s_{n} \sim n^{2 / d_{s}}
$$

is the crossover time at which the ant first recognizes that it does not move on the infinite cluster, but on a finite one with $n$ sites, i.e. the time it needs to travel a distance that is comparable to the cluster's extension. For times $s \gg s_{n}$, on the other hand, every site in the cluster is equally likely to be visited by the ant, hence $P^{(n)}(s) \sim 1 / n$ in this case. Taken together, we can write

$$
P^{(n)}(s) \sim s^{-d_{s} / 2} f_{2}\left(s / s_{n}\right)+1 / n
$$

for $s \gg 1$. The cut-off function $f_{2}(x)$ is of order one for $x \lesssim 1$ and decreases rapidly to zero for $x \rightarrow \infty$. Upon comparing (15) and (18), we infer the relation

$$
\left.\left\langle\frac{1}{n} \operatorname{Tr}\left[\left(1-E_{0}\right) \mathrm{e}^{-s \Gamma / b}\right]\right\rangle_{n}\right|_{\varepsilon=0} \sim s^{-d_{s} / 2} f_{2}\left(s / s_{n}\right)
$$

for $s \gg 1$. To derive (19) we have also used the explicit form $\left(E_{0}\right)_{i j}=n^{-1}, 1 \leq i, j \leq n$, of the matrix elements of the eigenprojector corresponding to the non-degenerate zero eigenvalue of $\Gamma$ for the case of a single cluster with $n$ sites.

\section{Critical behaviour of stress relaxation}

The long-time decay of the stress relaxation function $G$ is determined by the biggest clusters. Close to the gelation transition we may thus use the scaling form (12) of the cluster-size distribution and approximate the (Riemann) sum on the right-hand side of (10) by the corresponding integral

$$
G(t, \varepsilon) \sim\left(n^{*}(\varepsilon)\right)^{2-\tau} \int_{0}^{\infty} \mathrm{d} x x^{1-\tau} f_{1}(x) G_{x n^{*}(\varepsilon)}(t, \varepsilon) .
$$

If $(20)$ is to be consistent with the scaling form (1), we are forced to require the functional dependence

$$
G_{n}(t, \varepsilon) \sim t^{-a} f_{3}\left(n / n^{*}(\varepsilon), t / t^{*}(\varepsilon)\right) .
$$

The yet unknown exponent $a$ and the time scale $t^{*}(\varepsilon) \sim \varepsilon^{-z}$ can be determined from the limiting case $\varepsilon=0$,

$$
G_{n}(t, 0) \sim t^{-d_{s} / 2} f_{2}\left(t / t_{n}\right) \quad \text { with } \quad t_{n} \sim n^{2 / d_{s}},
$$

which is obtained from (11), (19) and (17). Indeed, for (22) to be the limit of (21) as $\varepsilon \downarrow 0$, one has to identify $a=d_{s} / 2$, and the diverging $\varepsilon$-dependencies in $f_{3}$ have to cancel each other in order to yield a non-trivial limit. This is only possible if $f_{3}(x, y) \sim f_{2}\left(y / x^{\alpha}\right)$ as $x, y \rightarrow 0$ with some exponent $\alpha$ which guarantees $t^{*}(\varepsilon) \sim\left(n^{*}(\varepsilon)\right)^{\alpha}$. Consequently, we also get the relation $t_{n} \sim n^{\alpha}$. Hence, $\alpha=2 / d_{s}$ by $(22)$, and we conclude $t^{*}(\varepsilon) \sim \varepsilon^{-z}$ with $z=2 /\left(d_{s} \sigma\right)$. Moreover, expressing $n^{*}(\varepsilon)$ in terms of $t^{*}(\varepsilon)$, the stress-relaxation function (20) takes on the form

$$
G(t, \varepsilon) \sim t^{-\Delta}\left(t / t^{*}(\varepsilon)\right)^{\Delta-d_{s} / 2} \int_{0}^{\infty} \mathrm{d} x x^{1-\tau} f_{1}(x) f_{3}\left(x, t / t^{*}(\varepsilon)\right)
$$


for $\varepsilon \ll 1$ and $t \rightarrow \infty$ with $\Delta=(\tau-1) d_{s} / 2$. This completes the derivation of (4).

\section{Discussion and outlook}

Table 1 summarizes the exact critical exponents for stress relaxation in the Rouse model for gelling polymers below and at the gel point. The exponents differ from the predictions $k=2 \nu-\beta$ and $\Delta=d \nu /(d \nu+k)$ of earlier scaling arguments $[6,7,10,14,16,35]$. What is the reason for this discrepancy? The scaling arguments involve the Hausdorff fractal dimension $d_{f}:=d-\beta / \nu$ of rigid percolation clusters at $c_{\text {crit }}$. Rouse clusters, however, are thermally stabilized, Gaussian phantom clusters with the Hausdorff fractal dimension

$d_{f}^{(\mathrm{G})}:=2 d_{s} /\left(2-d_{s}\right)[15,31,33]$, which is different from $d_{f}$ in space dimensions below the upper critical dimension $d_{u}=6$. Indeed, if one replaces $d_{f}$ by $d_{f}^{(\mathrm{G})}$ in these scaling arguments, as one should consistently do in a Rouse description, the results will coincide with Table 1.

Since the scaling relations $k=2 \nu-\beta$ and $\Delta=d \nu /(d \nu+k)$ involve the Hausdorff fractal dimension $d_{f}$ of rigid percolation clusters, it is sometimes argued that they describe the behaviour of a more realistic model, which, in addition to the interactions of the Rouse model, accounts for excluded-volume effects, too, see e.g. [35]. To the best of the author's knowledge this claim has not been verified yet by strict analytical arguments within a microscopic model. One may even have doubts whether this claim is generally true: Extensive molecular-dynamics simulations [26] of a system of crosslinked soft spheres in three dimensions, with a cluster ensemble as in the present Paper and an additional strongly repulsive interaction at short distances, yield the values $k \approx 0.7$ and $\Delta \approx 0.75$, which are remarkably close to the predictions of the Rouse model for gelling polymers, see Table 1 . On the other hand, simulations of the bond-fluctuation model in [25] imply $k \approx 1.3$ and are thus in favour of the claim. However, the viscosity is not measured directly in these latter simulations. Rather it is derived from the scaling of diffusion constants and an additional scaling assumption that may be questioned [26]. Hence, it is an open problem to what extent the critical exponents of Table 1 are modified by excluded-volume interactions.

\section{Acknowledgments}

My sincere thanks go to Henning Löwe and Annette Zippelius for both enlightening discussions and a critical reading of the manuscript. Partial financial support by SFB 602 of the Deutsche Forschungsgemeinschaft is acknowledged.

\section{References}

[1] Stauffer D, Coniglio A and Adam M 1982 Adv. Polym. Sci. 44103

[2] Daoud M and Lapp A 1990 J. Phys. Cond. Matter 24021

[3] Martin J E and Adolf D 1991 Annu. Rev. Phys. Chem. 42311

[4] Winter H H and Mours M 1997 Adv. Polym. Sci. 134165 
[5] Adam M, Delsanti M, Durand D, Hild G and Munch J P 1981 Pure Appl. Chem. 531489

[6] Durand D, Delsanti M, Adam M and Luck J M 1987 Europhys. Lett. 3297

[7] Martin J E, Adolf D and Wilcoxon J P 1988 Phys. Rev. Lett. 612620

[8] Adolf D and Martin J E 1990 Macromolecules 233700

[9] Devreux F, Boilot J P, Chaput F, Malier L and Axelos M A V 1993 Phys. Rev. E 472689

[10] Colby R H, Gillmor J R and Rubinstein M 1993 Phys. Rev. E 483712

[11] Vlassopoulos D, Chira I, Loppinet B and McGrail P T 1998 Rheol. Acta 37614

[12] Tordjeman P, Fargette C and Mutin P H 2001 J. Rheol. 45995

[13] Stauffer D 1976 J. Chem. Soc. Faraday Trans. II $\mathbf{7 2} 1354$

[14] de Gennes P-G 1978 Comptes Rendus Acad. Sci. (Paris) 286B 131

[15] Cates M E 1984 Phys. Rev. Lett. 53926

Cates M E 1985 J. Physique (France) 461059

[16] Martin J E, Adolf D and Wilcoxon J P 1989 Phys. Rev. A 391325

[17] Rubinstein M, Zurek S, McLeish T C B and Ball R C 1990 J. Physique (France) 51757

[18] Broderix K, Goldbart P M and Zippelius A 1997 Phys. Rev. Lett. 793688

[19] Zilman A G and Granek R 1998 Phys. Rev. E 58 R2725

[20] Broderix K, Löwe H, Müller P and Zippelius A 1999 Europhys. Lett. 48421

[21] Broderix K, Löwe H, Müller P and Zippelius A 2001 Phys. Rev. E 6301151

[22] Broderix K, Aspelmeier T, Hartmann A K and Zippelius A 2001 Phys. Rev. E 64021404

[23] Broderix K, Löwe H, Müller P and Zippelius A 2001 Physica A 302279

[24] Broderix K, Müller P and Zippelius A 2002 Phys. Rev. E 65041505

[25] Del Gado E, de Arcangelis L and Coniglio A 2000 Eur. Phys. J. E 2359

[26] Vernon D, Plischke M and Joós B 2001 Phys. Rev. E 64031505

[27] Ferry J D 1980 Viscoelastic Properties of Polymers 2nd ed (New York: Wiley)

[28] Rouse P E 1953 J. Chem. Phys. 211272

[29] Doi M and Edwards S F 1986 The Theory of Polymer Dynamics (Oxford: Clarendon Press)

[30] Bird R B, Curtiss C F, Armstrong R C and Hassager O 1987 Dynamics of Polymeric Liquids vol 2, 2nd ed (New York: Wiley)

[31] Vilgis T A 1988 Physica A 153341

[32] Sommer J-U, Schulz M and Trautenberg H L 1993 J. Chem. Phys. 987515

[33] Sommer J-U and Blumen A 1995 J. Phys. A: Math. Gen. 286669

[34] Blumen A and Jurjiu A 2002 J. Chem. Phys. 1162636

[35] Rubinstein M, Colby R H and Gillmor J R 1989 Space-Time Organization in Macromolecular Fluids ed F Tanaka, M Doi and T Ohta (New York: Springer)

[36] Stauffer D and Aharony A 1994 Introduction to Percolation Theory revised 2nd ed (London: Taylor and Francis)

[37] Bunde A and Havlin S 1996 Fractals and Disordered Systems ed A Bunde and S Havlin (Berlin: Springer) pp 59, 115

[38] Alexander S and Orbach R 1982 J. Physique (France) 43 L-625

[39] de Gennes P-G 1979 J. Physique (France) Lett. 40, L-197

[40] Haan S W and Zwanzig R 1977 J. Phys. A: Math. Gen. 101547

[41] de Gennes P-G 1976 La Recherche 7919

[42] Havlin S and Ben-Avraham D 2002 Adv. Phys. 51187 